\title{
Cfd Simulation Of Swirling Effect In S-Shaped Diffusing Duct By Swirl Angle 20
}

\author{
Ramazan \\ Assistant Professor, Mechanical Engineering, Bearys Institute of Technology, Mangalore, India
}

\begin{abstract}
The present study involves the CFD analysis for the prediction of swirl effect on the characteristics of a steady, incompressible flow through an S-shaped diffusing duct. The curved diffuser considered in the present case has $S$ shaped diffusing duct having an area ratio of 1.9, length of $300 \mathrm{~mm}$ and turning angle of $22.5^{\circ} / 22.5^{\circ}$. The static pressure, total pressure, velocity and turbulence intensity were accounted.

The improvement is observed for both, clockwise and anti-clockwise swirl, the improvement being higher for clockwise swirl. Flow uniformity at the exit is more uniform for clockwise swirl at the inlet.
\end{abstract}

Keywords: Curved diffusers, intake ducts, swirling flow, secondary flows, pressure recovery

\section{INTRODUCTION}

Diffusers are one of the standard challenges in fluid mechanics. The task of a diffuser is to decelerate the flow and to regain total pressure. It is more difficult to arrange for an efficient deceleration of flow than it is to obtain an efficient acceleration ${ }^{5}$. There is a natural tendency in a diffusing process for the flow to break away from the walls of the diverging passage reverse its direction and flow back in direction of the pressure gradient. If the divergence is too rapid, this may result in the formation of eddies with consequent transfer of some kinetic energy into internal energy and a reduction in useful pressure rise.

\section{LITERATURE REVIEW}

The flow development in curved ducts with inflexion in curvature without diffusion has been reported by Rowe ${ }^{1}$ [1970]. The experiments were carried out in S-shaped ducts of mild curvature with the angle of turn being $45^{\circ} / 45^{\circ}$ and $30^{\circ} / 30^{\circ}$. He showed that in a $45^{\circ} / 45^{\circ} \mathrm{S}$-bend in which the secondary flow causes a complete interchange of wall fluid and fluid in the central core, possibly indicating the reason why heat transfer effects are large in wavy pipes.

Bradshaw $^{2}$ [1972] have studied the flow characteristics within an S-shaped duct with strong curvature. They measured only pressures and observed a pair of counter-rotating vortices at the exit. The ideal pressure recovery coefficient is derived directly from basic principles.
A computational investigation of flows in diffusing S-shaped ducts was provided by R.Menzies ${ }^{3}$. A high and low mass flow cases were compared with the experiments and calculations. The low mass flow case showed good comparison with experiment and calculations. A problem of the low mass flow case is the prediction of secondary flow. It was found that the SST turbulence model best predicted this feature.

Anand $^{4}$ [2001] studied on the effect of the turning angle on the flow and performance characteristics of long S-shaped circular diffusers. $\mathrm{He}$ established the effect of the turning angle on the flow and performance characteristics of long $S$-shaped circular diffusers (length-inlet diameter ratio, $\mathrm{L} / \mathrm{D}_{\mathrm{i}}=11.4$ having an area ratio of 1.9 and centre-line length of $600 \mathrm{~mm}$. The experiments are carried out for three S-shaped circular diffusers having angles of turn of $15^{\circ} / 15^{\circ}, 22.5^{\circ} / 22.5^{\circ}$ and $30^{\circ} / 30^{\circ}$.

A detailed experimental study was been carried out by Whitelaw and $\mathrm{Yu}^{5}$ [1993] on a scaled model of the RAE 2129 S-shaped intakediffusing duct having circular cross-section. The velocity and pressure characteristics of an Sshaped diffusing duct was measured in a water tunnel with three asymmetric inlet conditions using laser-Doppler anemometry at a Reynolds's number of 40,000 . The three inlet conditions were introduced by thickening the boundary layer at various locations along the inlet circumference and corresponding to a side-slip, zero angle of attack and low angle of attack. S-shaped duct is considered as an intake passage for jet aircraft. They found out that a presence of enlarged vortices had impaired the non-uniformity of the corresponding stream wise mean velocity distribution at the exit. The maximum secondary flow velocity however, did not exceed $15 \%$ of the inlet bulk velocity in all cases investigated.

\section{CFD ANALYSIS}

Computational Fluid Dynamics (CFD) can be described as the use of computers to produce information about the ways in which fluids flow in given situations. CFD embraces a variety of technologies including mathematics, computer science, engineering and physics, and these disciplines have to be brought together to provide the means of modeling fluid flows. Such modeling 
Ramazan / International Journal of Engineering Research and Applications

(IJERA) ISSN: 2248-9622 www.ijera.com

Vol. 3, Issue 4, Jul-Aug 2013, pp. $51-59$

is used in many fields of science and engineering but, if it is to be useful, the results that it yields must be a realistic simulation of a fluid in motion.

\section{PROPOSED WORK}

Ansys fluent is widely used for CFD analysis of various geometries. But In this study Sshaped diffuser is model created in ANSYS Design Modeller with the structured

multiblock mesh generation in ICEM CFD.

Simulations will be carried out in ANSYS FLUENT and appropriated boundary conditions for different faces were declared. The present study involves the CFD analysis for the prediction of swirl effect on the characteristics of a steady, incompressible flow through an S-shaped diffusing duct. The static pressure, total pressure, velocity and turbulence intensity were accounted.

\section{a) Numerical Modeling Approach}

\section{Simulation Procedure}

(STEP 1) Modeling (In ANSYS Design Modeller):

- Diffuser geometry is created by ANSYS Design Modeller using below dimentions.

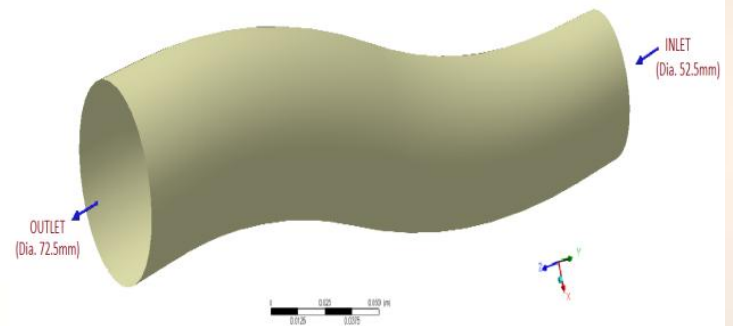

Fig. 1: Solid model of S-shaped diffusing duct

Table 1: Dimension of the model

\begin{tabular}{|l|c|}
\hline Inlet diameter & $52.5 \mathrm{~mm}$ \\
\hline Outlet diameter & $72.5 \mathrm{~mm}$ \\
\hline Area ratio & 1.9 \\
\hline Length of centre line & $300 \mathrm{~mm}$ \\
\hline Offset & $58.2 \mathrm{~mm}$ \\
\hline Turning angle & $22.5^{\circ} / 22.5^{\circ}$ \\
\hline Radius & $382 \mathrm{~mm}$ \\
\hline
\end{tabular}

The model is meshed in ICEM CFD.

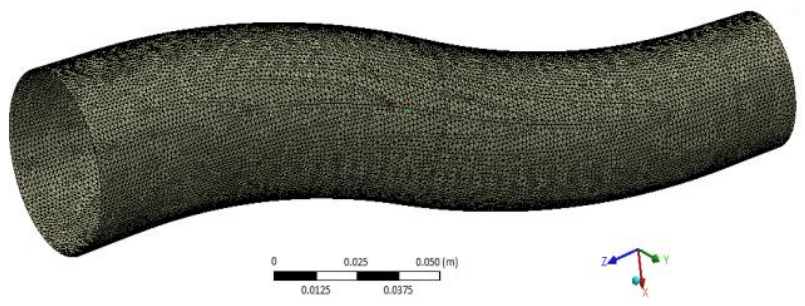

Fig. 2: Meshed model of test diffuser
- The model has been meshed with quadratic-mesh. Fine meshing with spacing 0.07 was done, mesh node's 245964 and mesh elements 229824.

- Boundary conditions taken were for velocity at inlet, pressure at outlet and wall type.

- Three simulations will be performed as per the experimental analysis with velocity magnitude of $27 \mathrm{~m} / \mathrm{s}$ :

1. Uniform flow at the inlet

2. Clockwise flow at the inlet (axial component $=\cos 20^{\circ} ;$ tangential component $=\sin 20^{\circ} ; \quad$ radial component $=0$ )

3. Anti-clockwise flow at the inlet (axial component $=\cos 20^{\circ} ;$ tangential component $=\quad-\sin 20^{\circ} ; \quad$ radial component $=0$ )

The operating conditions will be kept at atmospheric pressure with the outlet being exposed to it, i.e., pressure outlet. All the walls will be considered as smooth no-slip walls.

o Fluid was specified as air for the continuum type and the mesh was exported to Fluent for post processing.

(STEP 2) Post Processing (In ANSYS Fluent):

- Grid was checked and scaled.

- 3D solver and segregated solution method was chosen.

- Material property: Air was chosen as the fluid for flow, and its properties like density and viscosity at $25^{\circ} \mathrm{C}$ were selected.

- $\mathrm{k}-\omega$ SST model is selected for turbulence with Low Re-corrections with the first cell $y+$ being maintained less than or equal to 1 at all wall locations

- At air inlet section, the inlet velocity of 27 $\mathrm{m} / \mathrm{s}$ with different swirl intensity was specified.

- Turbulence intensity of 3\% based on inlet flow diameter was specified. At the exit section, the pressure was specified being equal to atmospheric pressure.

- Second order upwind scheme was selected to solve continuity and momentum equations.

○ Convergence criteria of $10^{-6}$ were taken.

- Solution was initialized at inlet and made to iterate until it converges.

Once solution is converged, various data for pressure and velocity were obtained and graphs were plotted.

V. RESULTS AND Discussion

Investigation of S-shaped diffuser has been 
Ramazan / International Journal of Engineering Research and Applications

(IJERA) ISSN: 2248-9622 Www.ijera.com

Vol. 3, Issue 4, Jul-Aug 2013, pp. $51-59$

carried out with help of FLUENT, a CFD tool to simulate the effect of swirl on the pressure recovery. The area ratio is kept constant at 1.9 . The velocity profile was of $27 \mathrm{~m} / \mathrm{s}$ with different swirling intensity as $0^{\circ}, 9^{\circ}, 18^{\circ}, 22.5^{\circ} / 4.5^{\circ}, 22.5^{\circ} / 13.5^{\circ}$ and $22.5^{\circ} / 22.5^{\circ}$.

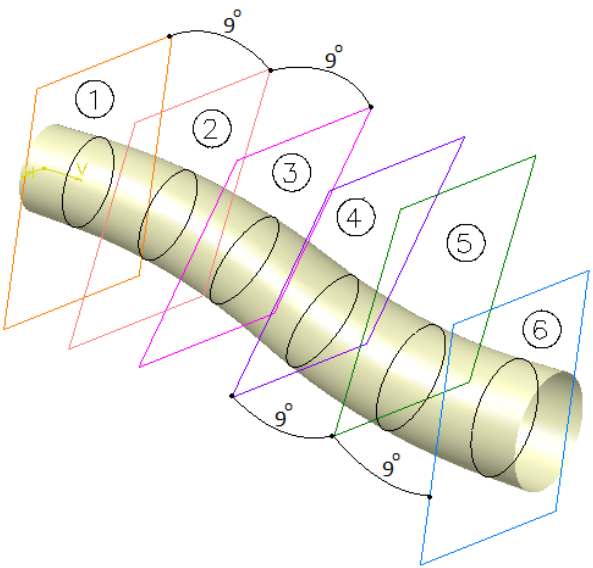

Fig. 3 Model showing planes and sections through which the parameters will be measured

Analysis gives the effect of swirl angle at flow separation and on pressure recovery coefficient.

\section{a) Graphs of Flow Separation \\ 1) Variation of Static Pressure Recovery Coefficient and Total Pressure Loss}

Table 3: Showing the values of $C_{p}$ and $C_{p . l o s s}$ for uniform and swirled flows at different sections uniform flow Clockwise-flow-20-deo Anti-Clockwise-flow-20-den

\begin{tabular}{|c|c|c|c|c|c|c|}
\hline & \multicolumn{2}{|c|}{ : } & \multicolumn{2}{|c|}{ 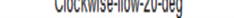 } & \multicolumn{2}{|c|}{ 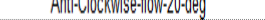 } \\
\hline Position-Section & Cp.uniform flow & Cploss-uniform flow & Cp-CW-2O-deg & Coloss-CW-20-deg & Cp-A-CW-2O-deg & Cp.loss-A.CW-2D-deg \\
\hline Section-0 & 0.086 & 0.0185 & 0.0938 & 0.0149 & 0.0938 & 0.0149 \\
\hline Section-9 & 0.232 & 0.0478 & 0.2440 & 0.0456 & 0.2445 & 0.0456 \\
\hline Section-18 & 0.325 & 0.0791 & 0.3594 & 0.0674 & 0.3594 & 0.0673 \\
\hline Section-22.544.5 & 0.299 & 0.1610 & 0.4482 & 0.0832 & 0.4481 & 0.0847 \\
\hline Section-22.5/13.5 & 0.389 & 0.1729 & 0.5188 & 0.0972 & 0.5162 & 0.1042 \\
\hline Section-22.5/22.5 & 0.487 & 0.1683 & 0.5721 & 0.1136 & 0.5699 & 0.1171 \\
\hline
\end{tabular}

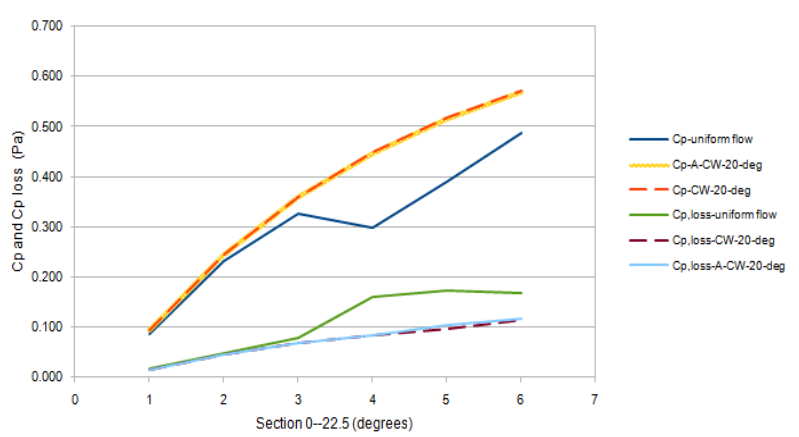

Fig. 4 Variation of performance parameters along diffusing duct

A) Uniform Flow Characteristics:

It is clear from the graph of Fig. 4 for a uniform flow at inlet:

1. Static pressure recovery coefficient: In the first bend, the mass-averaged static pressure recovery coefficient increases continuously and then suddenly gets reduced slightly near to the point of inflexion due to change of curvature. The recovery coefficient again picks up just after the inflexion due to flow diffusion as a result of increase in cross-sectional area.

2. Total pressure loss coefficient: The total pressure loss coefficient follows the same trend as that of the static pressure recovery coefficient.

3. The overall static pressure recovery and total pressure loss coefficients are 0.487 and 0.1683 of the inlet dynamic pressure $\left(0.5 \rho \mathrm{U}_{\text {long }}^{2}\right)$ respectively.

The ideal pressure recovery $\left(1-1 / \mathrm{AR}^{2}\right)$ for this diffuser should be $72.3 \%$. The lower pressure recovery compared with ideal static pressure recovery achieved may be due to the loss of inlet energy to creation of secondary flows and to overcome the higher surface resistance.

\section{B) Swirl Flow Characteristics:}

It is clear from the graph of Fig.4 for a swirl flow at inlet:

1. Static pressure recovery coefficient: The mass-averaged static pressure recovery coefficient increases gradually without getting affected by the inflexion in curvature. It is also observed that static pressure recovery coefficient for all the cases are comparatively much lower than the ideal recovery coefficient for this diffusing duct.

2. Total pressure loss coefficient: The overall total pressure loss coefficient also increases for the swirl flow, which indicates high shear.

3. The overall static pressure recovery and total pressure loss coefficients for both clockwise and anti-clockwise swirl are 0.5721 and 0.1136 of the inlet dynamic pressure $\left(0.5 \rho \mathrm{U}_{\text {long }}^{2}\right)$ respectively.

The increase of static pressure recovery could be due to the suppression of self-generated secondary flows as a result of swirl flow. It is observed that for both clockwise and anti-clockwise swirl flow, the overall static pressure recovery coefficient $(\sim 0.5721)$ is higher than that for uniform flow ( 0.487). The slightly higher recovery for clockwise/anti-clockwise indicates that the loss in energy for the uniform flow case is slightly higher as a result of swirling angles. Thus, in all the above cases the low value static pressure recovery coefficient as compared to the ideal pressure recovery coefficient $(\sim 0.723)$ could be due to the 


\section{Ramazan / International Journal of Engineering Research and Applications \\ (IJERA) ISSN: 2248-9622 www.ijera.com \\ Vol. 3, Issue 4, Jul-Aug 2013, pp. 51-59}

combined effect of curvature resulting in the generation of secondary flows and surface roughness of the diffusing duct walls.

\section{Longitudinal velocity distribution for uniform flow}

Figure 5a gives the normalized longitudinal velocity $\left(U_{\text {long }} / U_{\text {ave(in) }}\right)$ distribution at different sections for uniform flow. Figure 4a (i) confirms that the fluid flow at inlet section is almost uniform except near the wall. At section 2, the fluid core shifts towards the outer surface under the action of centrifugal force. This initial distortion is observed up to section 4 (Fig. 5a (ii)), i.e., just downstream of the point of inflexion. There is no abrupt change observed between sections 2 and 3 , and hence figures for those sections (section $2 \& 3$ ) are not presented here. The bulk flow retains its position in the second bend. However, there is a change in the direction of curvature: the maximum velocities are observed near to the convex surface and minimum near the concave surface of second bend. No further qualitative changes are observed in the velocity distribution until the exit (Fig. 5a (iii)) of the diffusing duct. At the end of the first bend, streamwise separation occurs very close to the inner surface of the first bend. The separated flow is seen from section 4 (Fig. 5a (ii)) and extends nearly up to the duct exit.

\section{Cross-flow velocity distribution for uniform flow} Normalized cross-flow velocity $\left(U_{\mathrm{sec}} / U_{\mathrm{ave}}(\mathrm{in})\right)$ vectors for different planes of the duct are given in Fig. 4b. It is seen that even at the inlet (Fig. 4b (i)) very weak secondary flows are observed which could be seen as an upstream effect of the downstream curvature. At downstream stations this intensity increases and a pair of secondary vortices is observed dividing the plane in nearly two halves (Fig. 4b (ii)), which is an expected trend due to the interaction between the low energy fluid collected near the inner surface (convex surface) and self-generated radial pressure gradient due to centrifugal force. In other words, these vortical motions may be the result of the imbalance of pressure driven secondary motions which forces the low-energy fluid from concave surface to convex surface along the circular wall. To maintain the continuity, the flow close to the convex surface moves towards the concave surface. Consequently, contra rotating vortical motions are developed near top and bottom surface. The vortices occupy almost 1/3rd portion of the exit cross-section (Fig. 4b (iii)). It is also seen that at the exit the vortices in the vortical pair are not equal in strength and size. There is slight reduction in the cross-flow velocity vectors at the exit in comparison to the plane just downstream of the inflexion.

\section{Turbulence intensity distribution for uniform flow \\ Normalized turbulence intensity} distribution across different sections of the duct is presented in Fig. 4c.From Fig. 4c (i), it can be seen that the turbulence intensity at inlet is low and fairly uniform. The turbulence intensity continuously increases at successive cross-sections along the duct length. As the flow moves into the diffuser the distortion of turbulence intensity profiles is minimized close to outer surface (Fig. 4c (ii)) of the first bend due to the bulk movement of fluid towards the outer surface under the effect of centrifugal force and self imposed pressure gradient.The intensity continuously increases further (Fig. 4c (iii)) close to outer surface of the second bend. The turbulence intensity at the exit of the duct is quite low $(\sim 22.069 \mathrm{j} / \mathrm{kg})$ for major portion of the crosssection. The high turbulence intensity can be seen at the exit to be concentrated at the central portion of the duct.

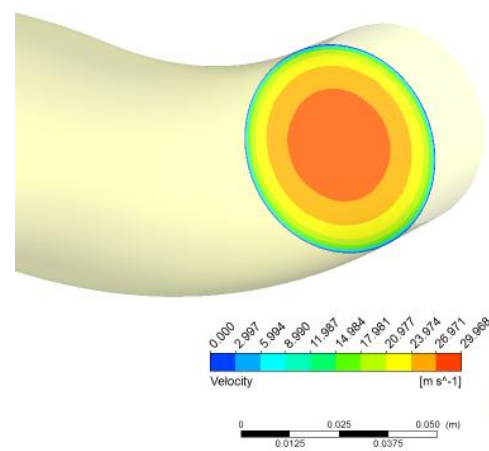

i) $0^{\circ}$

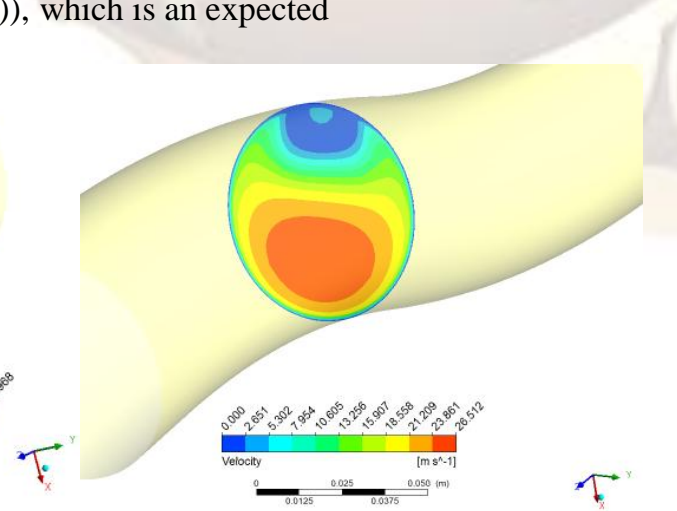

ii) $22.5^{\circ} / 4.5^{\circ}$ iii) $22.5^{\circ} / 22.5^{\circ}$

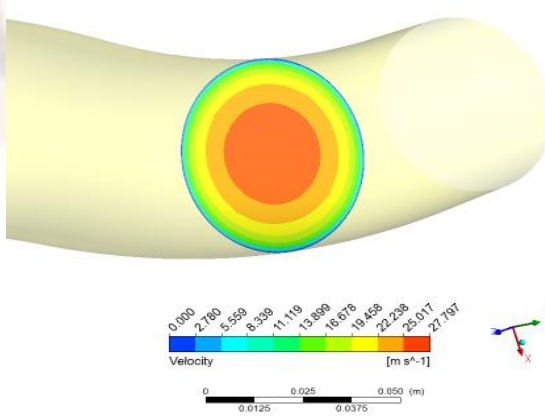

-

Fig. 5a-Normalized longitudinal velocity distribution with the uniform flow 


\section{Ramazan / International Journal of Engineering Research and Applications
(IJERA)
ISSN: 2248-9622
www.ijera.com

Vol. 3, Issue 4, Jul-Aug 2013, pp. 51-59

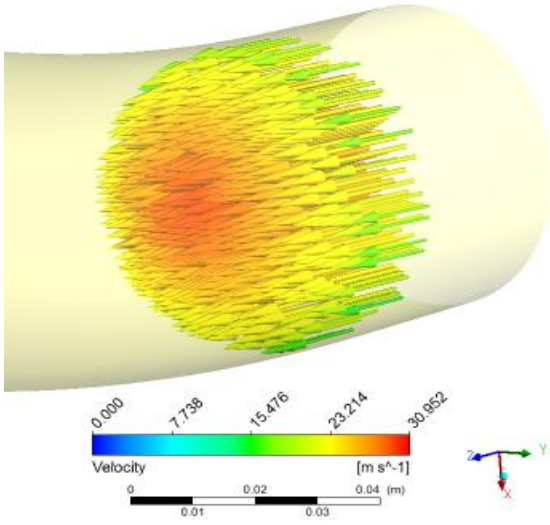

i) $0^{\circ}$

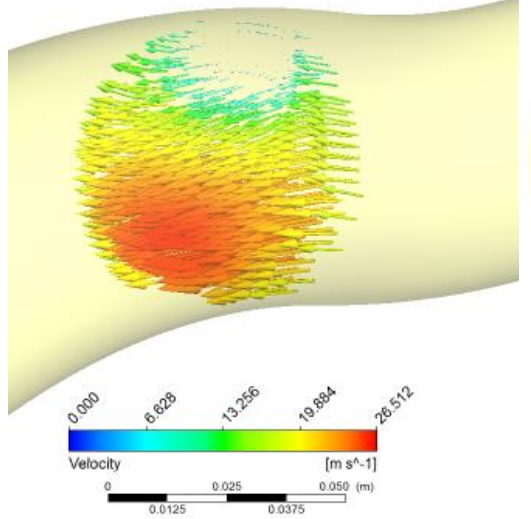

ii) $22.5^{\circ} / 4.5^{\circ}$

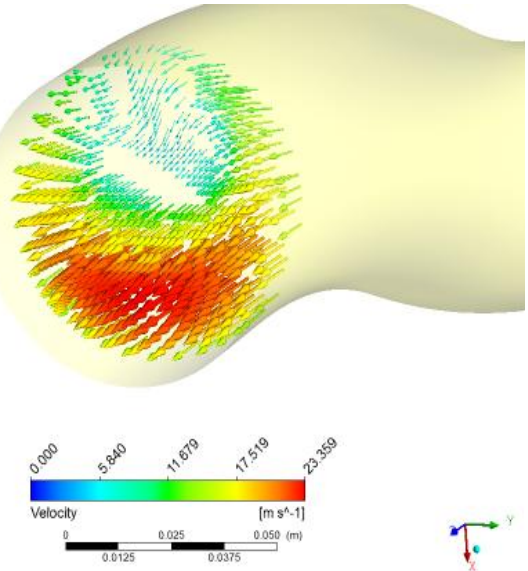

iii) $22.5^{\circ} / 22.5^{\circ}$

Fig. 5b-Normalized cross-flow velocity distribution with the uniform flow

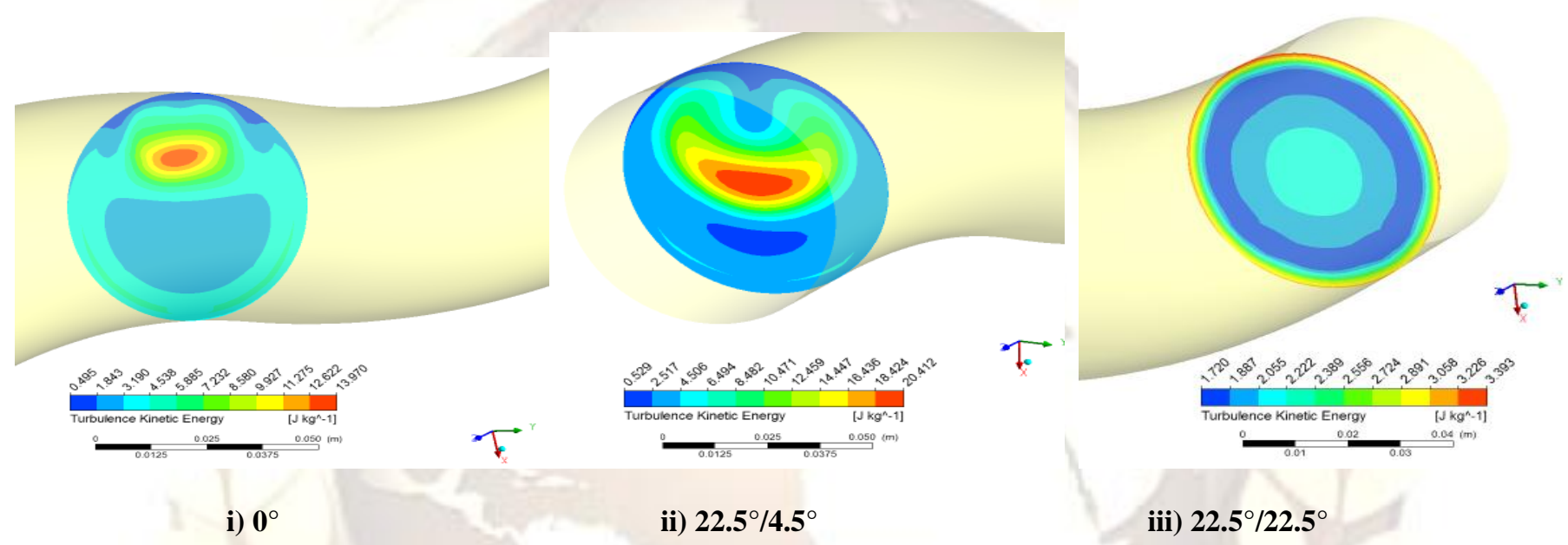

Fig. 5c-Turbulence intensity distribution with the uniform flow

\section{Longitudinal velocity distribution for clockwise} swirl

Figure $5 \mathrm{a}$ presents the normalized longitudinal velocity distribution at sections $0^{0}$, $22.50 / 4.5^{\circ}$ and $22.5^{0} / 22.5^{0}$ for clockwise swirl flow at the inlet.

At inlet, the major mass of fluid moves to the peripheral surface and there is fall in the center

due to the influence of the swirler. The combined effect of centrifugal force due to imposed swirl flow and geometry curvature makes the fluid move outwards. This can also be seen from Fig. 5a (ii). As the flow moves downstream, the same sense of rotation is observed until the exit (Fig. 5b (iii)). In comparison to the no swirl case, the bulk of flow has shifted from the convex wall to the top of the duct and it is comparatively more uniform.

\section{Cross-flow velocity distribution for clockwise swirl flow}

Normalized cross-flow velocity distribution at the same sections as above for clockwise swirl flow at inlet is shown in Fig. 5 b. From Fig. 5b (i), it can be seen that the cross-flow at inlet is completely rotational and no vortex pair is formed as seen for uniform flow at inlet. When the flow moves into the duct, there is a slight increase in the magnitude of cross-flow velocity in the top portion of the duct, whereas in the bottom portion there is a reduction in cross-flow velocity. This can be observed from Fig. 5b (ii). In addition, it is also noticed at the inlet that the cross-flow velocity core is slightly shifted outwards due to the downstream curvature. The values of the crossflow velocities decrease in successive downstream sections. The center of the swirling core slightly shifts towards the concave surface in the first bend. The same sense of rotation continues in the second bend. The effect of swirl continues to be observed until the diffuser exit (Fig. 5a (iii)). In the second bend, due to a change in direction of curvature, the cross-flow fluid core starts moving towards concave surface of the second bend (Fig. 5a (iii)). From the cross-flow velocity distribution, it is seen that the intensity of inlet swirl is high enough not to allow the formation of the pair of vortices as seen 


\section{Ramazan / International Journal of Engineering Research and Applications}
(IJERA)
ISSN: 2248-9622
www.ijera.com

\section{Vol. 3, Issue 4, Jul-Aug 2013, pp. 51-59}

for no swirl case. The cross-flow velocity shows clear circular motion across the planes in the clockwise direct

\section{Turbulence intensity distribution for clockwise swirling}

The corresponding normalized turbulence intensity variation at the same sections for clockwise swirl flow at inlet is shown in Fig. 5c. At inlet (Fig. 5c (i)), intensity values are higher than those observed for uniform flow velocity (Fig. 4c (i). However, as the flow moves downstream, the intensity magnitudes undergo a gradual reduction, and finally at the exit (Fig. 5c (iii)) the values are much lower than those observed for uniform flow velocity (Fig. 4c (iii)). This may be due to suppression of the secondary flows as a result of interaction between swirl flow and centrifugal force.

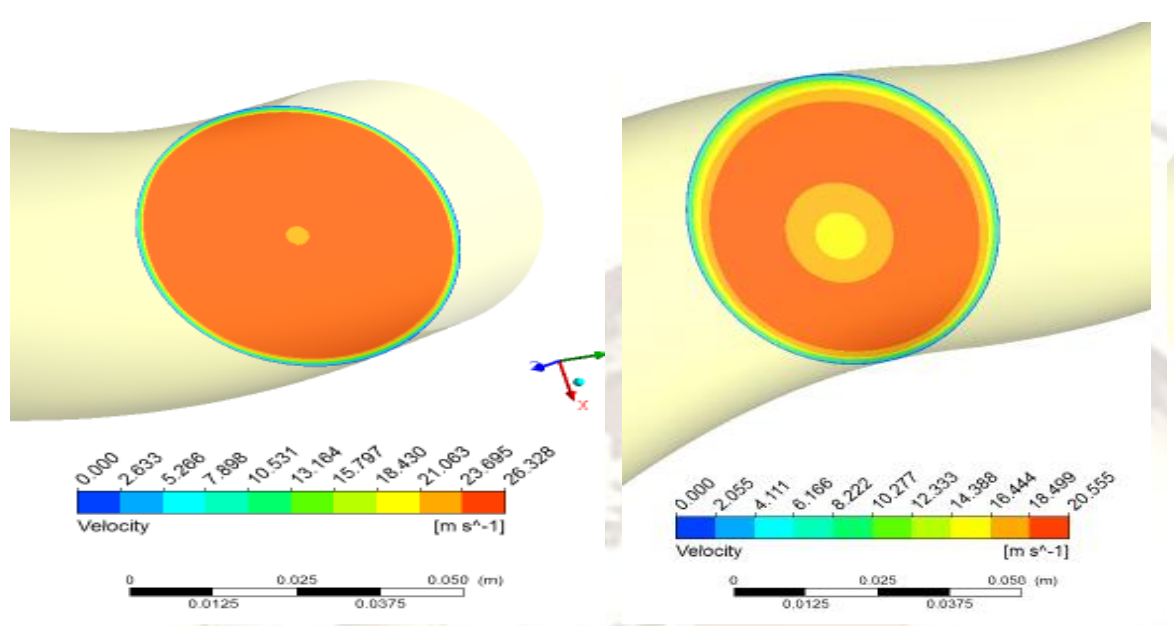

ii) $22.5^{\circ} / 4.5^{\circ}$
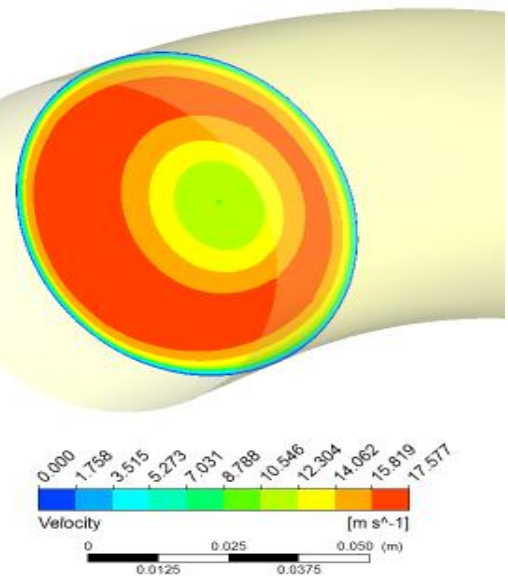

iii) $22.5^{\circ} / 22.5^{\circ}$

Fig. 5a-Normalized longitudinal velocity distribution with clockwise swirl

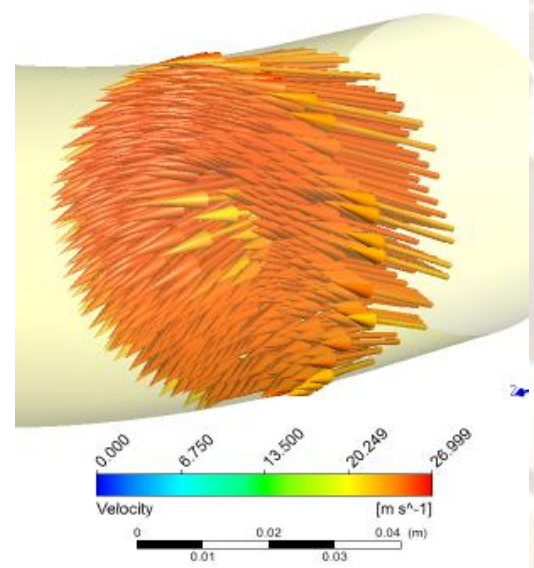

i) $0^{\circ}$

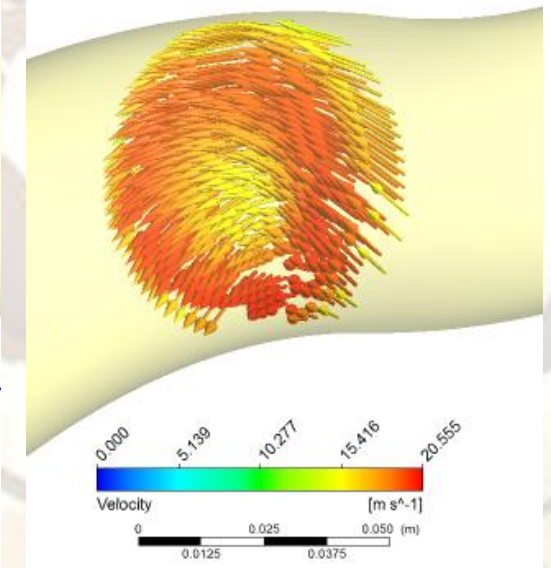

ii) $22.5^{\circ} / 4.5^{\circ}$

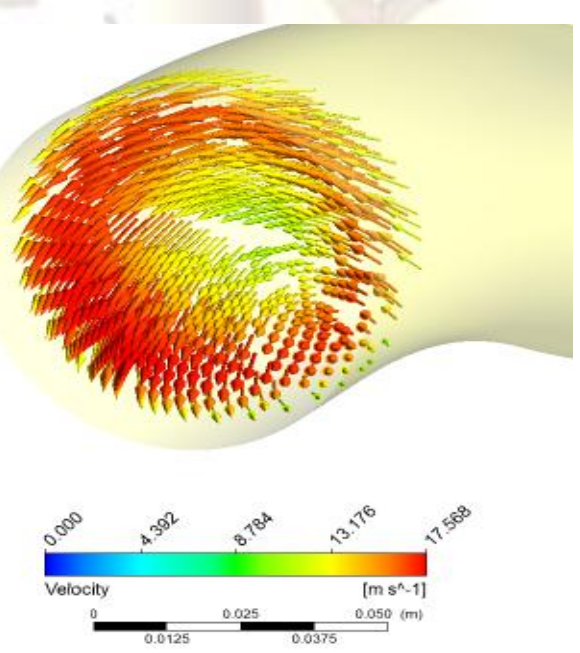

iii) $22.5^{\circ} / 22.5^{\circ}$

Fig. 5b-Normalized cross-flow velocity distribution with clockwise swirl 


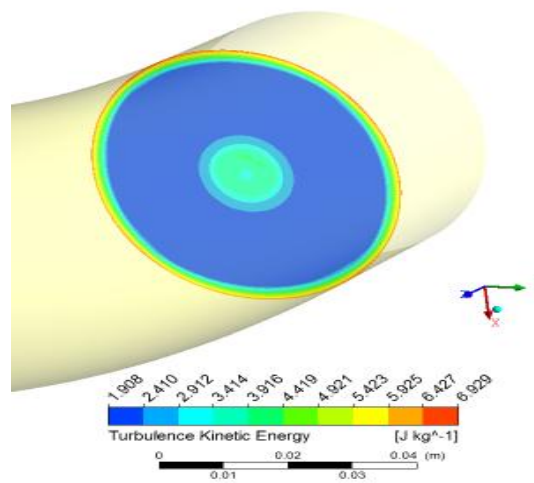

i) $0^{\circ}$

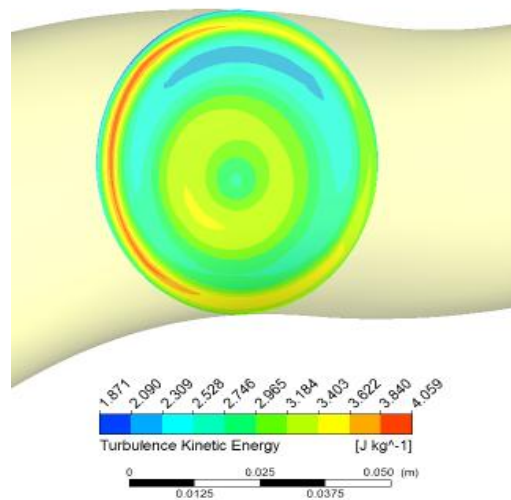

ii) $22.5^{\circ} / 4.5^{\circ}$

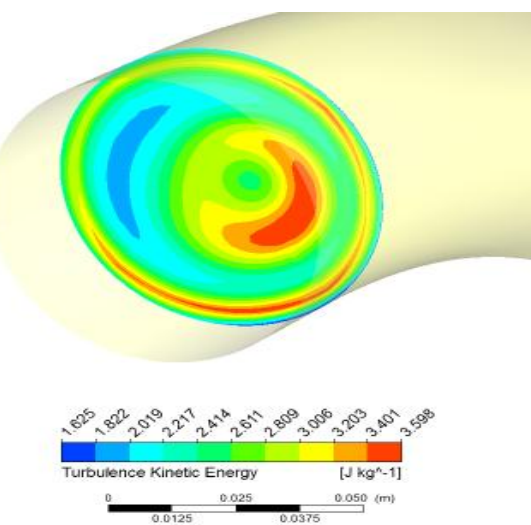

iii) $22.5^{\circ} / 22.5^{\circ}$

Fig. 5c-Turbulence intensity distribution with clockwise swirl flow

\section{Longitudinal Velocity Distribution for Anti-} Clockwise Swirl

The normalized longitudinal velocity distributions for anti-clockwise swirl flow at inlet are shown in Fig 6a (i). The following observations and conclusions can be drawn from the respective figures:

a) On comparison with clockwise swirl flow there is no major qualitative difference in velocity distribution.

The minor deviations observed is the bulk flow at the exit for anti-clockwise has moved towards the bottom instead of top and the velocity magnitude is maximum for anti-clockwise as compared to clockwise swirl flow. This implies that clockwise swirl flow at inlet leads to a more uniform flow at exit.

\section{Cross-flow velocity distribution for Anti- Clockwise Swirl}

The cross-flow distributions for anticlockwise swirl flow at inlet are shown in $7 \mathrm{i}$ Error! Reference source not found.. The following observations and conclusions can be drawn from the respective figures:

a) On comparison with clockwise swirl flow there is no major qualitative difference in cross-flow velocity distribution.

The minor deviations observed is that cross-flow velocity core is slightly shifted towards the concave wall where as for clockwise it was towards convex wall.

Turbulence Intensity Distribution for AntiClockwise Swirl

The turbulence intensity distributions for anti-clockwise swirl flow at inlet are shown in Fig. 6c. The following observations and conclusions can be drawn from the respective figures:

a) On comparison with clockwise swirl flow there is no major qualitative difference in turbulence intensity distribution.

The minor deviations observed is that turbulence intensity at the exit is relatively higher for anticlockwise swirl compared to clockwise swirl but is still lower than that for uniform flow.

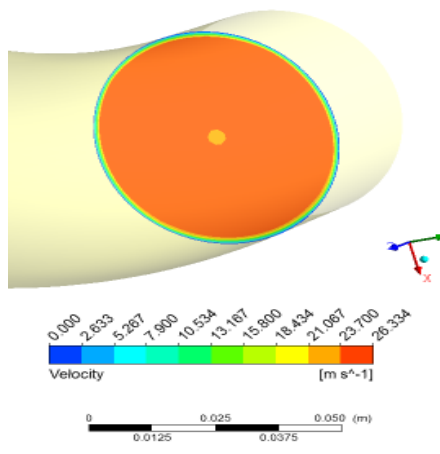

i) $0^{\circ}$

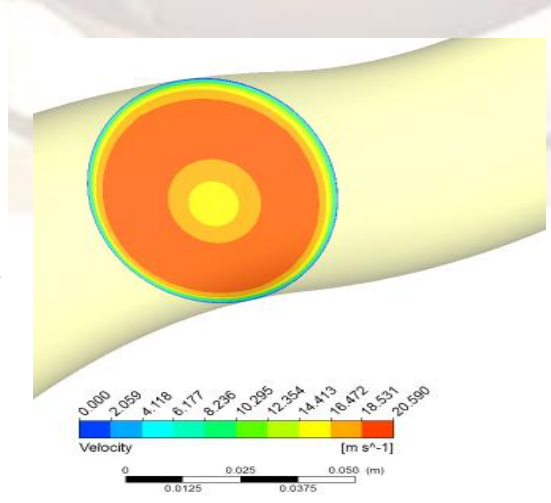

ii) $22.5^{\circ} / 4.5^{\circ}$

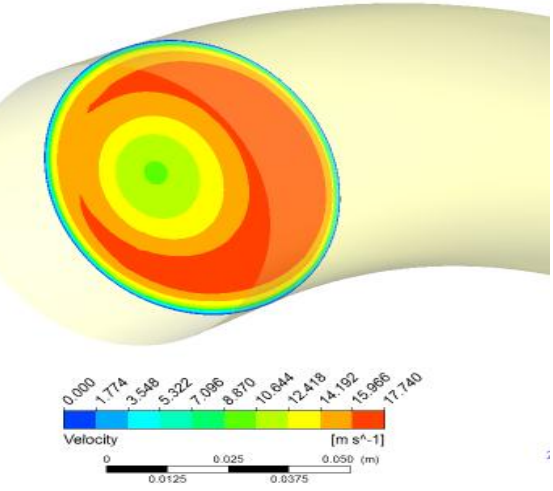

iii) $22.5^{\circ} / 22.5^{\circ}$

Fig. 6a-Normalized longitudinal velocity distribution with anti-clockwise swirl 


\section{Ramazan / International Journal of Engineering Research and Applications}
(IJERA)
ISSN: 2248-9622
www.ijera.com

\section{Vol. 3, Issue 4, Jul-Aug 2013, pp. 51-59}

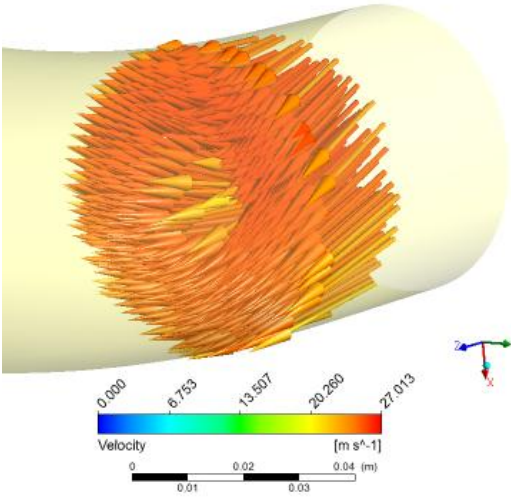

i) $0^{\circ}$

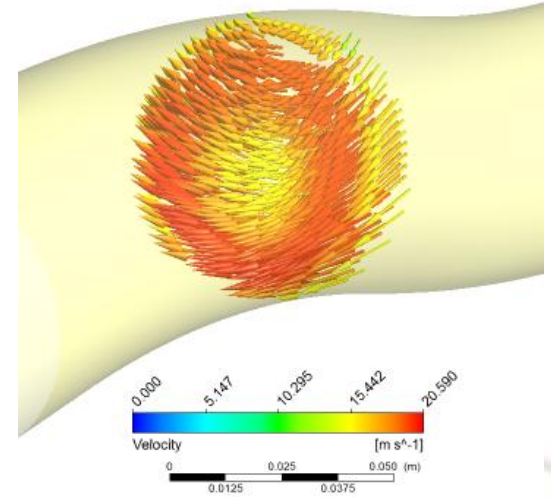

ii) $22.5^{\circ} / 4.5^{\circ}$

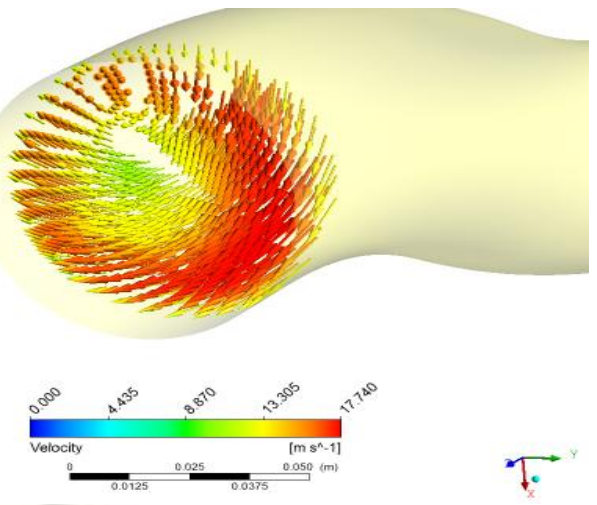

iii)

Fig. 6b-Normalized cross-flow velocity distribution with anti-clockwise swirl

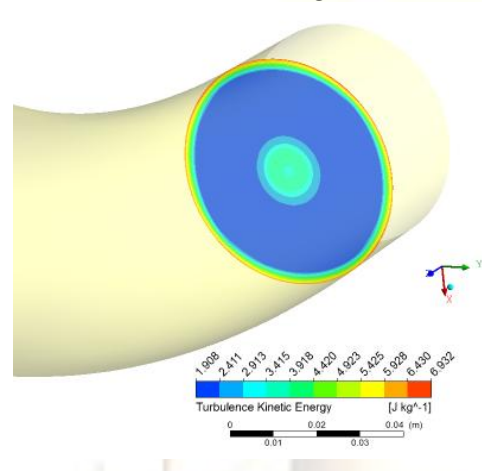

i) $0^{\circ}$

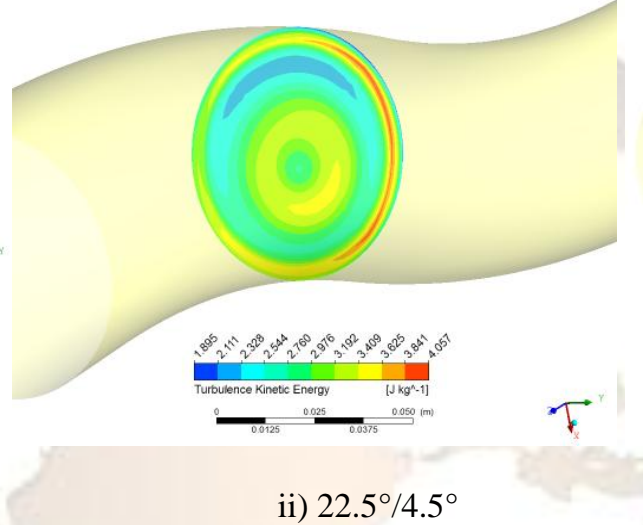

ii) $22.5^{\circ} / 4.5^{\circ}$
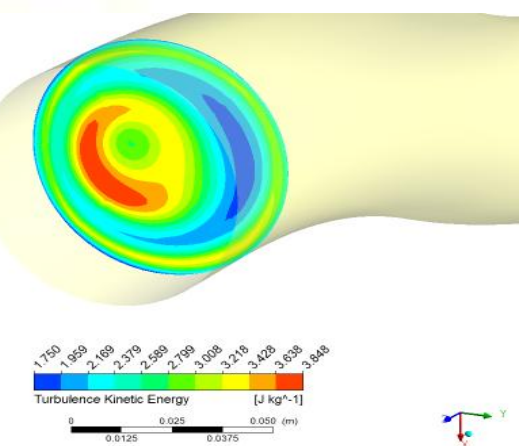

iii) $22.5^{\circ} / 22.5^{\circ}$

Fig. 6c-Turbulence intensity distribution with anti-clockwise swirl

\section{CONCLUSION}

On the basis of analysis and results presented, the following conclusion can be drawn:

- The overall static pressure recovery increases for swirl flow at inlet irrespective of direction of swirl (clockwise or anticlockwise), the increase being around $40 \%$. The loss coefficient also increases for swirl flow.

- The flow distribution at the exit plane is more uniform for clockwise swirl in comparison to anti-clockwise swirl and uniform flow at inlet.

- The secondary flow is present throughout the diffuser for uniform flow. A pair of vortices is observed at all the sections with the intensity of the two vortices being not equal. For swirl flow, only circular motion is present and no pair of vortices is formed.

- Turbulence intensity variation indicates the presence of high turbulence intensity at the planes just before and after the inflexion plane. Turbulence intensity variation at the exit for the clockwise swirl flow is much lower compared to the uniform flow at the inlet.

\section{REFERENCE}

1] M.ROWE, "Measurements and computations of flow in pipe bends", J. Fluid Mech., JFM (Printed in GReat Britain), vol. 43, part 4, pp. 771-783, 1970.

2] Bansod P, \& Bradshaw P, Aeronautical Q, 23 (1972) 131-140.

3] R.Menzies, "Computational investigations of flows in diffusing S-shaped intakes", Acta Polytechnica ,Vol. 41 No. 4-5/2001

4] Anand R B, Lajpat Rai, Singh S N \& Sharma," Effect of the turning angle on the flow and performance characteristics of long S-shaped circular diffusers", Journal of Aerospace Engineering, JAE (Professional Engineering Publishing Ltd), 53 (2001) 239-252.

5] Whitelaw J H \& Yu S C M, Flow Meas Instrum, 4 (1993), 171-179.

6] Guo R W \& Seddon J, Aeronaut Q, 33 (1982) 25-58.

7] Guo R W \& Seddon J, Aeronaut Q, 34 (1983) 99-129. 


\section{Ramazan / International Journal of Engineering Research and Applications \\ (IJERA) ISSN: 2248-9622 www.ijera.com \\ Vol. 3, Issue 4, Jul-Aug 2013, pp. $51-59$}

8] Majumdar B, Singh S N \& Agrawal D P, Proc Inst Mechanical Eng, (Pt A), 210 (1996) 65-75.

9] Anand R B, Lajpat Rai, Singh S N \& Sharma O P, J Aero Soc India, 53 (2001) 239-252.

10] Cockrell, D.J., Markland, E., 1963. “A Review of Incompressible Diffuser Flow" Aircraft Engg. Volume 35, pp 286.

\section{AUTHORS:}

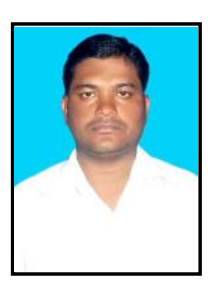

Ramazan is currently working as Assistant professor in Mechanical Engineering, in BIT, Mangalore affiliated to Vishveshvarayya Technical University of Karnataka State (India). He received his M Tech from PDA Gulbarga and B.E. Degree from M.V. Jayaramanan College of Engineering. Bangalore form Vishveshvarayya University, Belgaum (India), in 2007. He is actively engaged in teaching since 3 years and has guided good number of projects at the graduate level. His areas of interest in CFD Analysis of aerospace components and as well as in Nano technology. 